\title{
Evidence for preferential pairing in telotrisomic plants of rye
}

\author{
Elena Benavente and \\ Juan Orellana
}

\begin{abstract}
Departamento de Genética, Facultad de Biología, Universidad Complutense, 28040 Madrid, Spain
\end{abstract}

Using C-banding it has been possible to distinguish all meiotic configurations at metaphase I in telotrisomic plants of rye. From these configurations the types of pairing among the short arms of chromosome $1 R$, in trisomic condition, could be directly established. The results indicate that there are actually pairing preferences and they could be responsible for the low trivalent frequencies found. The long arm of chromosome $1 \mathrm{R}$, in disomic condition, seems to play an important role on pairing preferences which shows the short one. The possibility of pref erences produced by chiasma formation and chiasma failures is also discussed.

\section{INTRODUCTION}

When more than two chromosome arms are present at meiosis, a competitive situation exists between potential pairing partners. Most attempts to study pairing preferences in aneuploids and polyploids have been made from the analysis of meiotic configurations at metaphase $\mathrm{I}$, with conventional staining techniques (John and Henderson, 1962; Timmis and Rees, 1971 ; Sybenga, 1975; Elçi and Sybenga, 1976; Jackson and Hauber, 1982; Chapman, 1984; Callow et al., 1984). Due to the impossibility of distinguishing specific chromosome arms with such techniques, pairing preferences have been indirectly determined by using theoretical models often based on severe restrictions such as assuming that,

1. all chromosomes of different sets and all chromosomes within the same set have the same probability of pairing. This is reasonable only for very special materials such as isogenic tetraploids newly derived from successively doubled haploids (Callow et al., 1984).

2. the existence of only one pairing initiation point located at the end of each chromosome arm. Then, multivalents at pachytene must be twice as often as bivalents or univalents if pairing is at random. Deviations from this ratio have been taken as evidence of preferential pairing. However, the existence of several synaptic initiation points per arm would lead to multivalent frequencies higher than $2 / 3$ because partner exchanges might occur more frequently as it has been reported by John and Henderson (1962), Sybenga (1975) and Callow et al. (1984).

There are some discrepancies concerning the meiotic configurations which should be used for the approach. Timmis and Rees (1971) and Chapman (1984) only used those configurations with complete pairing in autotetraploid rye, because the remaining ones, in which any arm is not bound, could represent larger configurations that had degradated by chiasma failures.

In contrast, Sybenga's model (1975) takes account of all meiotic configurations at metaphase I to estimate the expected values of multivalent frequencies at pachytene, since multivalents, in general, have lower chiasma frequency than bivalents, and the selection of configurations with complete pairing automatically excludes a high frequency (potential) of multivalents.

It has been demonstrated in rye that the probability of being bound is different for the different chromosomes arms (see Orellana and Giraldez, 1983), possibly because there is a between arms variation with respect to the number of pairing initiation points (Abirached-Darmency et al., 1983). Therefore the mere deviation from $2 / 3$ of multivalents should not be taken as an evidence of pairing preferences.

In this paper we present clear evidence for preferential pairing in telotrisomic plants of rye, directly analysed at metaphase I by using a Cbanding technique. 


\section{MATERIAL AND METHODS}

Four telotrisomics for the short arm of chromosome 1R were used for this study.

All telotrisomic plants of rye appeared spontaneously in the offsprings obtained from the crosses between a $1 \mathrm{R}$ telocentric substitution line of cv. Petkus Spring (kindly supplied by Dr Sybenga, Agric. Univ., Wageningen, The Netherlands) and one plant of inbred line E (Giraldez et al., 1979) (tE6) or one plant of Secale cereale ssp segetale ( $\mathrm{tSeg} 7, \mathrm{tSeg} 11, \mathrm{tSeg} 17)$.

To obtain mitotic metaphase cells, seeds were germinated on wet filter paper in Petri dishes at room temperature. When primary roots were 1 $2 \mathrm{~cm}$ long they were excised and immersed in tap water at $0^{\circ} \mathrm{C}$ for 48 hours to shorten the chromosomes. Subsequently, the tips were fixed in acetic alcohol $1: 3$. For meiotic cells, anthers were fixed in acetic alcohol $1: 3$. The fixed material was squashed and stained following the Giesma Cbanding described previously (Giraldez et al, 1979).

\section{RESULTS}

Using the C-banding technique it is possible to identify specific chromosome arms of rye in mitosis as well as in meiosis due to the presence of prominent blocks of $\mathrm{C}$-heterochromatin in most of the telomeres (see Orellana and Giraldez, 1984).

At metaphase I, for a chromosome in telotrisomic condition the following meiotic configurations can be expected: frying pan trivalent $(F$, with partner exchange and interstitial and distal chiasmata in the trisomic arm in combination with chiasmata in the other arm); Y-shaped trivalent ( $Y$, with partner exchange and interstitial and distal chiasmata in the trisomic arm, but not accompanied by chiasmata in the other arm); chain trivalent $(\mathrm{C})$; ring bivalent + telo univalent $\left(\mathrm{R}+\mathrm{I}^{\mathrm{t}}\right)$; open bivalent, in which the long or the short arm is bound, + telo univalent $\left(\mathrm{O}_{1}+\mathrm{I}^{\mathrm{t}}\right.$ or $\mathrm{O}_{\mathrm{s}}+\mathrm{I}^{\mathrm{t}}$, respectively); heteromorphic bivalent + univalent $(\mathrm{H}+\mathrm{I})$ and univalent triplet (U). Fig. 2 shows some samples of these configurations.

The plants of cv. Petkus Spring used in the crosses showed a C-banding pattern for the chromosome $1 \mathrm{R}$ characterised by the presence of prominents blocks of $\mathrm{C}$-heterochromatin in the telomeres of both arms of the standard chromosome, as well as in the $1 \mathrm{R}^{\mathrm{S}}$ telocentric chromosome. The plant of inbred line E did not show such bands in its telomeres and the plant of $S$. cereale $s s p$

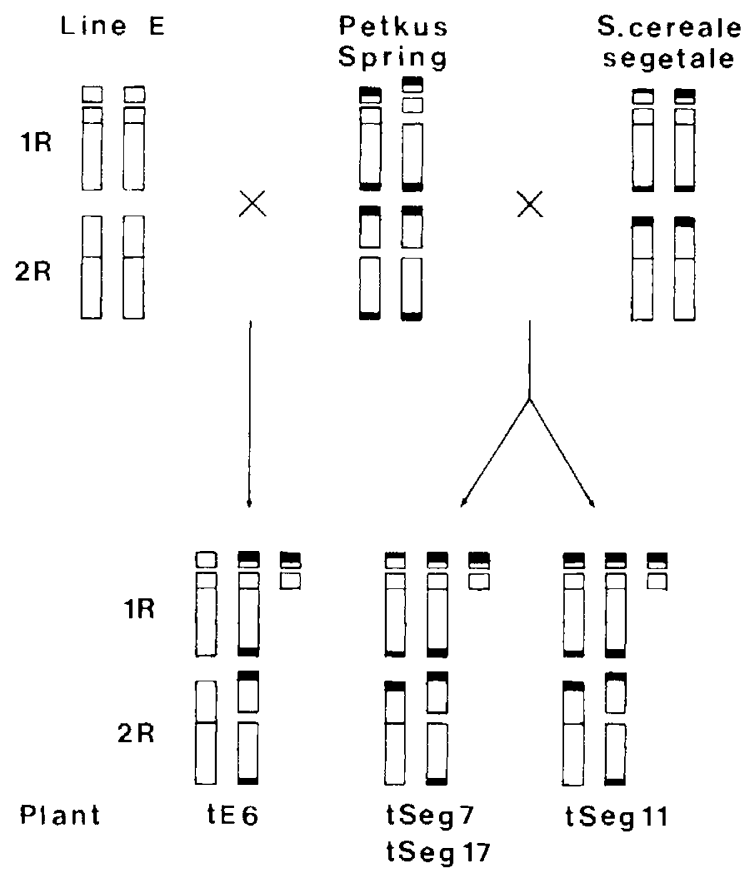

Figure 1 C-banding pattern for chromosomes $1 R$ and $2 R$ of the four telotrisomic plants of rye analysed.

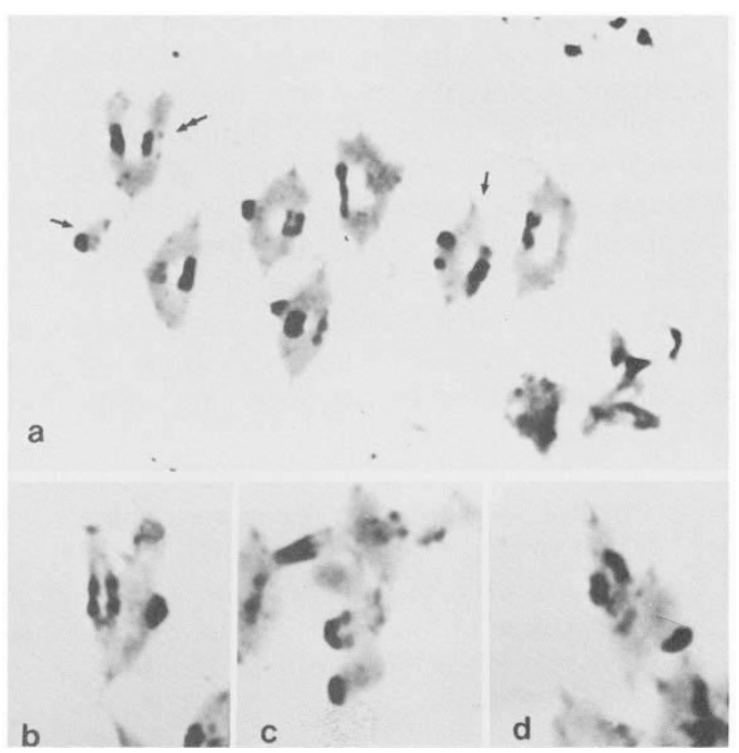

Figure 2 Different meiotic configurations for chromosome $1 \mathrm{R}$ in telotrisomic plants of rye. (a) C-banded metaphse 1 cell of plant tSeg11; arrows indicate one $1 \mathrm{R}$ ring bivalent with S-S (Pet-Seg) type of pairing for both arms and one telocentric univalent; double arrow indicates one U-shaped trivalent for chromosome $2 \mathrm{R}$. (b) chain trivalent with Pet-t type of pairing for the short arm. (c) open bivalent in which the short arm is bound with Pet-E type of pairing. (d) frying pan trivalent. 
segetale showed different amount of C-heterochromatin in the short arms and the same in the long ones of both $1 \mathrm{R}$ homologues (see fig. 1).

When more than two arms are present in the same plant pairing competition can occur. This is the situation of the telotrisomics of rye analysed here.

The C-heterochromatin blocks and the telocentric chromosome used as cytological markers have allowed us to study, directly, pairing preferences at metaphase I since it has been possible to distinguish the different types of pairing for the arm in trisomic condition.

Plant tE6 possesses two 1R standard chromosomes, one from Petkus Spring (Pet) and the other from inbred line $E(E)$, and one $1 R^{S}$ telocentric chromosome $(\mathrm{t})$ from Petkus Spring. Then, in this plant, three types of pairing could be observed for the $1 R^{S}$ chromosome arm: Pet-E, E-t and Pet-t (figs. $2 b-c$ ).

In the same way, plants tSeg7, tSeg11 and tSeg 17 possess two $1 \mathrm{R}$ standard chromosomes, one from Petkus Spring (Pet) and the other from segetale $(\mathrm{Seg})$ and one $1 \mathrm{R}^{\mathrm{S}}$ telo $(\mathrm{t})$. In $\mathrm{tSeg} 7$ and tSeg17 three types of pairing (Pet-Seg, Seg-t and Pet- $t$ ) could be distinguished due to the presence of a thin C-band in the short arm of chromosome $1 \mathrm{R}$ from segetale. However, such $1 \mathrm{R}^{\mathrm{S}}$ chromosome arm shows, in $\mathrm{tSeg} 11$, a big heterochromatic block very similar to the one of Petkus Spring and, consequently, only two types of pairing involving standard or standard and telocentric chromosomes (S-S, S-t) could be differentiated (fig. 2a). In all plants, when more than two arms were associated at the same point (as in frying pan and $\mathrm{Y}$-shaped trivalents) the type of pairing was considered undetermined (fig. 2d).

The heterozygosity for C-bands makes it possible to distinguish two types of chain trivalents (C) and heteromorphic bivalents $(\mathrm{H})$ since the telo could be associated either with Petkus or E/Seg chromosomes.

It is worth mentioning that all the plants analysed here have one chromosome $2 \mathrm{R}$ substituted by its corresponding telos (telocentric substitution individuals) since the Petkus Spring parents were homozygous ditelocentric metacentric for $2 \mathrm{R}$.

Table 1 shows the frequencies of the different meiotic configurations observed at metaphase $I$ in all plants analysed.

According to Sybenga (1975) if there is only one point of pairing initiation per arm, and in absence of pairing preferences a ratio of $2: 1$ trivalents: bivalents could be expected in organisms with pronounced distal chiasmata localisation. Table 2 shows the trivalent frequencies observed and expected under these assumptions. In all plants there is an excess of bivalents, the differences being highly significant.

A trivalent frequency lower than $2 / 3$ could be explained by the existence of preferential pairing. This hypothesis was tested in our material since it was possible to estimate the frequencies of the different pairing types by using the C-banding technique.

Assuming random pairing, a ratio $1: 1: 1$ Pet-E, E-t, Pet-t for tE6 and Pet-Seg, Seg-t, Pet-t for tSeg7 and $\mathrm{tSeg} 17$ types of pairing is expected. However, the expected values S-S, S-t for tSeg11 must be $1: 2$. Table 3 shows the comparison between the observed and expected values under this assumption. The highly significant differences found indicate the existence of pairing preferences between the three arms.

On the other hand, late diplotene cells (fig. 3) were analysed in plants tE6 and tSeg17 (table 4). In both cases the frequencies of trivalents were higher at this stage than at metaphase I.

Table 1 Frequencies of the different meiotic configurations ( $F$, frying pan trivalent; $C$, chain trivalent; $\mathrm{R}$, ring bivalent; Ol, open bivalent with the long arm bound; Os, open bivalent with the short arm bound; $H$, heteromorphic bivalent $; I$, standard univalent; $I^{t}$, telocentric univalent ; $\mathrm{U}$, univalent triplet) for chromosome $1 \mathrm{R}$ observed at metaphase $\mathrm{I}$ in the telotrisomic plants of rye

\begin{tabular}{|c|c|c|c|c|c|c|c|c|c|c|}
\hline \multirow{3}{*}{$\frac{\text { Plant }}{\text { tE6 }}$} & \multicolumn{9}{|c|}{ Meiotic configurations } & \multirow{3}{*}{$\frac{\text { No. cells }}{550}$} \\
\hline & \multirow{2}{*}{$\begin{array}{l}F \\
3\end{array}$} & \multicolumn{2}{|c|}{$\mathrm{C}$} & \multirow{2}{*}{$\frac{\mathrm{R}+\mathrm{I}^{\mathrm{t}}}{114}$} & \multirow{2}{*}{$\frac{\mathrm{OI}+\mathrm{I}^{\mathrm{t}}}{311}$} & \multirow{2}{*}{$\frac{\mathrm{Os}+\mathrm{I}^{\mathrm{t}}}{2}$} & \multicolumn{2}{|c|}{$\mathrm{H}+\mathrm{I}$} & \multirow{2}{*}{$\begin{array}{l}\mathrm{U} \\
-\end{array}$} & \\
\hline & & $71^{*}$ & $43+$ & & & & $2^{*}$ & - & & \\
\hline $\operatorname{tSeg} 7$ & 3 & $33^{*}$ & $74+$ & 77 & 111 & $\ldots$ & - & $1 末$ & 1 & 300 \\
\hline $\operatorname{tSeg} 17$ & 6 & $4^{*}$ & $55 \ddagger$ & 101 & 130 & - & $1^{*}$ & $1 \neq$ & 2 & 300 \\
\hline $\operatorname{tSeg} 11$ & 4 & & & 69 & 106 & 1 & 1 & & 5 & 250 \\
\hline
\end{tabular}

\footnotetext{
* With Pet-t type of pairing for the short arm.

$\dagger$ With E-t type of pairing for the short arm.

$\ddagger$ With Seg-t type of pairing for the short arm.
} 
Table 2 Comparison between observed and expected trivalent frequencies at metaphase $I$ assuming random pairing $(2: 1$ trivalent, bivalent)

\begin{tabular}{lllllll}
\hline Plant & & Triv. & Biv. & $X^{2}$ & & d.f. \\
\hline \multirow{2}{*}{ tE6 } & Obs. & 117 & 433 & 510.0191 & 1 & $\mathrm{p}<0.001$ \\
& Exp. & 366.67 & 183.33 & & & \\
\multirow{2}{*}{ tSeg7 } & Obs. & 110 & 190 & 121.50 & 1 & $\mathrm{p}<0.001$ \\
& Exp. & 200.00 & 100.00 & 121.50 & & \\
\multirow{2}{*}{ tSeg17 } & Obs. & 65 & 235 & 273.375 & 1 & $\mathrm{p}<0.001$ \\
& Exp. & 200.00 & 100.00 & & & \\
\multirow{2}{*}{ tSrg11 } & Obs. & 68 & 182 & 175.2474 & 1 & $\mathrm{p}<0.001$ \\
& Exp. & 166.67 & 83.33 & & & \\
\hline
\end{tabular}

\section{DISCUSSION}

In all plants analysed in this work a lower than $2 / 3$ trivalent frequency has been found (table 2 ). Similar results, as regards multivalent frequency, have been obtained in autotetraploid rye (Timmis and Rees, 1971; Chapman, 1984). But in these studies only ring quadrivalents and ring bivalents were used for the analysis. Meiotic configurations with low numbers of chiasmata were excluded because these configurations at metaphase I could have originated by chiasma failures either from quadrivalents and bivalents at pachytene.

Sybenga has pointed out (1975) that the exclusion of several meiotic configurations, such as chain quadrivalents and open bivalents, could be a source of error and, consequently, that this type of studies is not a good reflection of the meiotic behaviour at pachytene. He used a model in which all meiotic configurations at metaphase I are considered for estimating pairing frequencies at pachytene, but in this case the existence of chiasma failures could also lead to erroneous estimations.

Usually, the lack of fit between the observed and expected values calculated by the two ways has been explained by the existence of pairing preferences.

Table 3 Comparison between observed and expected frequencies of the different types of pairing for the short arm of chromosome $1 \mathrm{R}$ in telotrisomic plants. Expected values have been calculated by assuming random pairing (ratio $1: 1: 1$ for each type of pairing)

\begin{tabular}{|c|c|c|c|c|c|c|c|}
\hline \multirow[b]{2}{*}{ Plant } & & \multicolumn{3}{|c|}{ Types of pairing } & \multirow[b]{2}{*}{ Total } & \multirow[b]{2}{*}{$\mathrm{X}^{2}$} & \multirow[b]{2}{*}{ d.f. } \\
\hline & & Pet-E & E-t & Pet-t & & & \\
\hline \multirow[t]{2}{*}{ tE6 } & Obs. & 116 & 43 & 72 & 231 & $\begin{array}{l}35 \cdot 09 \\
n<0 \cdot 0001\end{array}$ & 2 \\
\hline & & \multicolumn{3}{|c|}{ Types of pairing } & & & \\
\hline \multicolumn{2}{|l|}{ Plant } & Pet-Seg & Seg-t & Pet-t & Total & $X^{2}$ & d.f. \\
\hline \multirow{2}{*}{ tSeg7 } & Obs. & 77 & 75 & 33 & 185 & $20 \cdot 02$ & 2 \\
\hline & Exp. & $61 \cdot 67$ & $61 \cdot 67$ & $61 \cdot 67$ & & $p<0.001$ & \\
\hline \multirow{2}{*}{$\operatorname{tSeg} 17$} & Obs. & 101 & 56 & 5 & 162 & $85 \cdot 44$ & 2 \\
\hline & Exp. & $54 \cdot 0$ & $54 \cdot 0$ & $54 \cdot 0$ & & $p<0.001$ & \\
\hline \multirow[b]{2}{*}{ Plant } & & \multicolumn{3}{|c|}{ Types of pairing } & & & \\
\hline & & $S-S$ & & & Total & $\mathrm{X}^{2}$ & d.f. \\
\hline \multirow{2}{*}{$\operatorname{tSeg} 11$} & Obs. & 70 & \multirow{2}{*}{\multicolumn{2}{|c|}{$\begin{array}{l}65 \\
90 \cdot 0\end{array}$}} & 135 & 20.83 & 1 \\
\hline & Hxp. & $45 \cdot 0$ & & & & $p<0.001$ & \\
\hline
\end{tabular}

Table 4 Frequencies of the different meiotic configurations for chromosome $1 \mathrm{R}$ observed at late diplotene in plants tF.6 and tSeg17. The mean number of $1 R$ trivalents per cell at this stage and at metaphase $I$ are also included

\begin{tabular}{|c|c|c|c|c|c|c|c|c|c|c|}
\hline \multirow[b]{2}{*}{ Plant } & \multicolumn{6}{|c|}{ Meiotic configurations } & \multicolumn{4}{|c|}{ Trivalents/cell } \\
\hline & $F$ & $\mathrm{C}$ & $\mathrm{R}+\mathrm{I}^{\prime}$ & $\mathrm{Ol}+\mathrm{I}^{\mathrm{t}}$ & $\mathrm{Os}+1^{\mathrm{t}}$ & $\mathrm{H}+\mathrm{I}$ & $\mathrm{U}$ & No. cells & Diplotene & Metaphase I \\
\hline tE6 & 9 & 43 & 36 & 19 & 1 & 1 & - & 109 & 0.477 & $0 \cdot 213$ \\
\hline tSeg17 & 22 & 34 & 34 & 10 & - & - & - & 100 & 0.560 & $0 \cdot 217$ \\
\hline
\end{tabular}




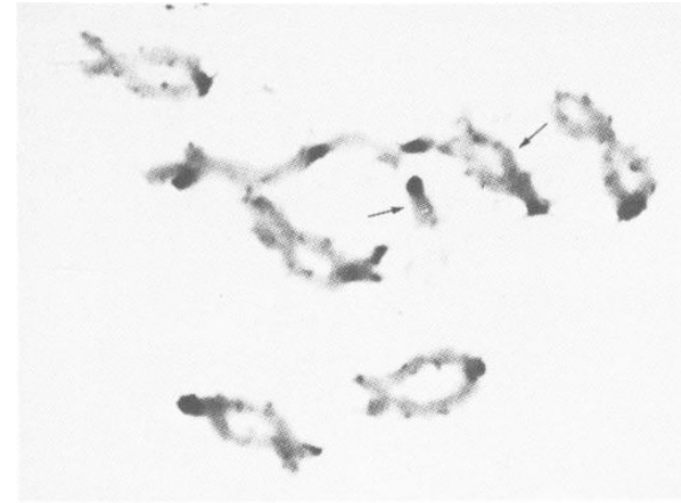

Figure 3 C-banded late diplotene cell of plant tE6; arrows indicate one ring bivalent and one telocentric univalent for chromosome $1 \mathrm{R}$.

On the other hand, with conventional staining techniques it is impossible to identify each specific set and pairing preferences are detected indirectly as a deviation from $2 / 3$ using the mean number of multivalents formed for all sets. This fact can account an additional source of error.

For triploids, primary trisomics and telotrisomics similar models can be used (Sybenga, 1975), but in these situations, the configurations with only complete pairing cannot be used due to the even number of chromosomes for each set and, consequently, all meiotic configurations at metaphase I must be considered. For this reason we have used the relation $2: 1$ trivalents: bivalents + univalents considering all metaphase I configurations according to Sybenga (1975).

A lower than $2 / 3$ trivalent frequency at metaphase I could be explained either by pairing preferences or by failures in chiasma formation.

Using C-banding technique, we have been able to analyse the different types of pairing for the arm in trisomic condition. The existence of pairing preferences is clear from the results of table 3 . The type of pairing involving the two standard chromosomes (Pet-E or Pet-Seg) is the most frequent in all plants analysed. Such pairing preferences would lead to a low trivalent frequency.

The models described above assume only one pairing initiation point per arm. However, the number and distribution of the pairing initiation points in diploid rye have been determined by Abirached-Darmency et al. (1983) from electron microscope observations of pollen mother cells at middle or late zygotene stages. These authors have indicate that pairing is often initiated from telomeric regions but also occurs at several sites along the chromosomes. They have found that about 6 and 13 sites exist in the short and long arms of chromosome $1 \mathrm{R}$, respectively. This might lead to differences in the timing of pairing initiation between both arms of chromosome $1 \mathrm{R}$.

The high probability for pairing of the long arm (in disomic condition) could facilitate the progress of pairing along the chromosome and consequently the frequency of pairing between the short arms of standard chromosomes would be increased. As observed in table 1, the probability of being bound for the long arm is very close to 1 and this fact would lead to an artificial pairing preference betwen these short arms, but not a pairing preference per se.

These pairing influences between both arms could be a general event in polyploids with metacentric or submetacentric chromosomes. In contrast, with telocentric chromosomes this problem is avoided. Thus, the tr'ce pairing preferences in telotrisomic plants $\mathrm{mL}_{\mathrm{L}} \mathrm{t}$ be estimated from the pairing frequencies of the telocentric chromosome with the two different standard ones. With random pairing a ratio $1: 1$ Pet-t, E-t/Seg-t is expected. In all cases the values were very different although no test was performed. It is worth mentioning that these pairing preferences are not related to the amount of C-heterochromatin.

The pairing preferences found either for the telocentric or standard chromosomes could be due to preferences in pairing or chiasma formation since the observations were made at metaphase I. Late diplotene cells were observed in plants tE6 and tSeg17 for resolving, in part, this problem (table 4). In all cases the frequencies of frying pan and chain trivalents were higher at this stage than at metaphase I. These results indicate the existence of a bound arm loss during meiotic prophase by chiasma failures, in disagreement with those of Maguire (1965) who observed the same trivalent frequency at pachytene as at metaphase $I$ in maize. This bound arm loss is not due to chiasma terminalisation because univalents or open bivalents with evidence of recombination have never been observed at metaphase I.

The number of associations at diplotene could be taken as a reflection of meiotic pairing at earlier stages when synapsis occurs, because arms associated at this stage have to derive from arms which have been synapsed previously. It is evident that many of these associations are non-chiasmate, and they are lost from diplotene to metaphase I leading to a decrease of trivalents at the later stage $(55 \cdot 3$ per cent for tE6 and $61 \cdot 25$ per cent for tSeg17) (see table 4). Probably, trivalent frequencies at pachytene or early diplotene would be higher, but, 
unfortunately, rye meiotic configurations are not clear at these stages. So, the trivalent frequencies estimated at late diplotene indicate that, perhaps, they could be very close to $2 / 3$ at earlier stages. However, this does not imply, necessarily, random pairing because the telocentric chromosome might be always paired with only one specific standard chromosome.

Associations at metaphase I can be taken as chiasmata. As it has been shown above, pairing as not at random at least at this stage, but we cannot discern if the preferences detected here are due either to preferences in chiasma formation with random pairing at pachytene or to pairing preferences at pachytene and a further chiasma formation at random.

Pairing preferences in a competitive pairing situation such as in polyploids and aneuploids have been explained by the existence of limited number of specific loci (zygomeres) (Sybenga, 1966) or by the different number of repeated pairing units that determine the pairing strength (Doyle, 1979). The variation in the number and localisation of such synaptic initiators per chromosome would lead to differences in pairing efficiency and activity. However, Giraldez and Santos (1981) in grasshoppers and Santos et al. (1983) in rye detected pairing preferences between identical and homologous chromosomes in diploid/tetraploid chimeras that could not be explained by differences in activity or efficiency but by differences in pairing affinities. Preferential pairing detected for the telocentric chromosome in this work could be explained by both hypotheses.

In summary, the telotrisomic plants of rye analysed here show lower than $2 / 3$ trivalent frequencies accompanied by pairing preferences at metaphase I between the three $1 \mathrm{R}^{\mathrm{S}}$ chromosome arms. These preferences cannot be ascribed to pachytene pairing or chiasma formation and further analysis at meiotic prophase is necessary to determine the degree of influence of such mechanisms on pairing preferences detected at metaphase I.

Acknowledgements This work has been supported by a grant from the Comisión Asesora dc Investigación Científica y Técnica of Spain.

\section{REFERENCES}

ABIRACHED-DARMENCY, M., 7.ICKLER, D. ANI) CAUDERON, Y. 1983. Synaptonemal complex and recombination nodules in rye (Secale cereale). Chromosoma, 88, 299-306.

CALLOW, R. S., HAMEY, Y. AND PATTRICK, S. M. 1984. Pairing of identical chromosomes in an isogenic tetraploid. Heredity, 53, 107-111.

CHAPMAN, C. G. D. 1984. Does preferential pairing occur in Secale cereale $\times$ Secale montanum tetraploids hyhrids? Heredity, 52, 317-322.

DOYLE, G. G. 1979. The allotetraploidization of maize. Part 1: The physical hasis-differential pairing affinity. Theor. Appl. Genet., 54, 103-112.

HI.ÇI, S. AND SYBENGA, .I. 1976. Incomplete preferential pairing in a tetraploid Secale hybrid carrying translocations: multivalent configuration frequencies and marker segregation. Genetica, 46, $177-182$.

GirAl.deZ, R., CERMEÑ(), M. C. AND OREllanA, J. 1979. Comparison of C-banding pattern in the chromosomes of inbred lines and open pollinated varieties of rye Secale cereale L. Z. Pflanzenzuchtg., 83, 40-48.

GIRALDIZZ, R. AND SANTOS, J. I, 1981. Cytological evidence for preferences of identical over homologous but not identical meiotic pairing. Chromosoma, 82, 447-451.

JACKSON, R. C. AND HAUbER, D. P. 1982. Autotriploid and autotetraploid cytogenetic analysis: correction coefficients for proposed binomial models. Am. J. Bot., 69, 646-648.

JOHN, B. ANI) HENDERSON, S. A. 1962. Analysis and polyploidy in Schistocerca paranensis. Chromosoma, 13, 111-147.

MAGUIRE, M. P. 1965. The relationship of crossover frequency to synaptic extent at pachytene in maize. Genetics, 5!, 23-40.

ORELlanA, J. AND GIRAlijez, R. 1983. Metaphase I bound arms and crossing over frequency in rye. III. Non-chiasmate bonds in desynaptic plants. Heredity, 51, 383-394.

ORELLANA, J. ANI) GIRALDEZ, R. 1984. Metaphase I bound arms and crossing over frequency in rye. IV. Triticale-rye hybrids. Heredity, 52, 265-271.

SANTOS, J. L., OREI.LANA, J. AND GIRALDEZ, R. 1983. Pairing competition between identical and homologous chromosomes in rye and grasshoppers. Genetics, 104, 677-684.

SYIBIENGA, J. 1966. The zygomere as hypothetical unit of chromosome pairing initiation. Genetica, 37, 186-198.

SYBENGA, J. 1975. Meiotic configurations. Monograph on Theoretical and Applied Genetics. Springer, Berlin, Heidelberg, New York.

TIMMIS, J. N. AND REES, H. 1971. A pairing restriction at pachytene upon multivalent formation in autotetraploids. Heredity, 26, 269-275. 